\title{
Particle-in-Cell Simulation of Second and Third Harmonic Cavity Klystron
}

\author{
Victoria C. R. Hill, Graeme Burt, \\ David Constable, Chris Lingwood \\ Department of Engineering \\ Lancaster University \\ Lancaster, England
}

\author{
Chiara Marrelli \\ Accelerator Division \\ European Spallation Source ESS \\ ERIC \\ Lund, Sweden
}

\author{
Igor Syratchev \\ CERN \\ Geneva, Switzerland
}

\begin{abstract}
This paper outlines the results obtained from Magic software for the CSM_23 (Core Stabilization Method) klystron. This klystron implements the use of a second and third harmonic klystron to increase the efficiency. From the PIC simulation an efficiency of $78.1 \%$ was achieved.
\end{abstract}

Keywords - klystron; high efficiency; third harmonic; second harmonic; MAGIC 2D; AJDisk.

\section{INTRODUCTION}

The RF power consumption in the large scale future accelerators such as Compact Linear Collider (CLIC) and Future Circular Collider (FCC) $[1,2]$ will be at a level of 100 MW. This demand makes it very important to employ the technology capable of very efficient RF power generation. Klystron amplifiers are the most common RF power sources that are used in particle accelerators. The existing technology is able to deliver multi-MW level devices with efficiency around $70 \%$. Recently, the new bunching technology: Core Oscillation Method (COM) [3], has been developed. It was been reported that in PIC simulations such klystrons showed RF power production efficiency of $85 \%$ [4].

Achieving the high efficiency in a klystron requires full bunch saturation, a situation when all the elections populate the bunch leaving the anti-bunch empty [3]. In COM this is realized by using the non-monotonic bunching technique, when the peripheral electrons are approaching the bunch center monotonically and core of the bunch is experienced periodical oscillations. These oscillations are generated by alternating bunching and de-bunching forces coming from bunching cavities impedances and space charge of the bunch itself. However such a technology requires substantial length to achieve full bunch saturation.

In this paper we present the results of PIC simulations of the tube based on alternative Core Stabilization bunching Method (CSM). In CSM the bunch saturation length is significantly reduced by using the harmonic $\left(2^{\text {nd }}\right.$ and $3^{\text {rd }}$ ) cavities $[5,6]$. The integrated impedance of these cavities set provides least modulation to the bunch core, whilst the bunching forces are rapidly increased towards the bunch periphery. As a result the bunch saturation happens much faster than in COM method and core of the bunch does not have time to expand. This effect gave the name to the method. CSM is best suited to L-band, where the wavelength to the klystron beam tunnel radius ratio is large enough to maintain sufficient impedance of the harmonic cavities. The CSM 23 klystron with parameters similar to the COM 08_4_0_4 tube reported in [4] (see table 1) was first optimized using 1D klystron code AJDisk [7]. The tube layout and Applegate diagram are shown in Figure 1. The efficiency of $84.2 \%$ was obtained in this optimization. Total length of CSM_23 is $1.72 \mathrm{~m}$. It is 3.3 times shorter than COM $08 \quad 0404$.

TABLE I. CSM_23 KLYSTRON PARAMETERS

\begin{tabular}{|c|c|}
\hline Parameter & Value \\
\hline Operating Frequency $(\mathrm{MHz})$ & 800 \\
\hline Beam Voltage $(\mathrm{kV})$ & 133.85 \\
\hline Beam Current $(\mathrm{A})$ & 12.551 \\
\hline Perveance $(\mu \mathrm{K})$ & 0.256 \\
\hline
\end{tabular}

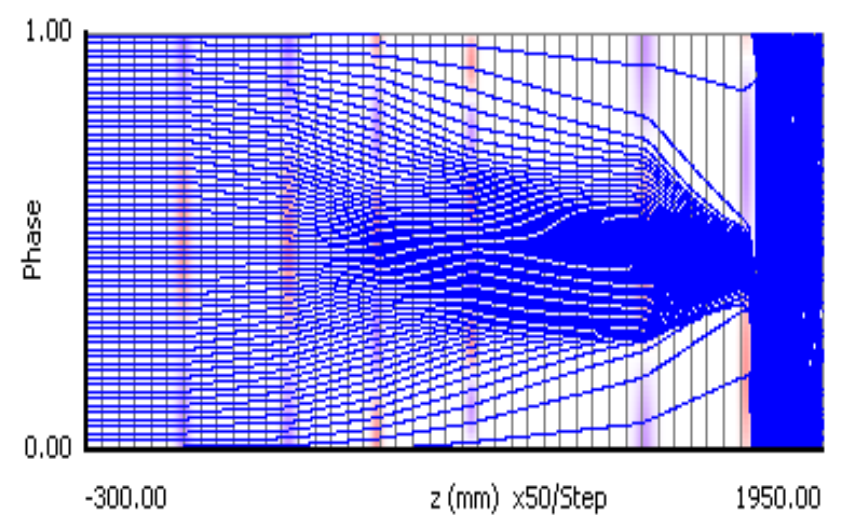

Figure 1. AJDisk Applegate diagram of CSM_23 klystron.

\section{PIC SIMULATION RESULTS}

First, on simulating CSM_23 in MAGIC2-D [8], the maximum output power of $1.31 \mathrm{MW}$ in saturation was achieved for an input power of $90 \mathrm{~W}$, corresponding to power gain of $41.6 \mathrm{~dB}$. For all input power levels considered, the output power is stable, with no reflected electrons predicted. The instantaneous bunch profiles in PZ-Z and R-Z phasespaces at the location of final two cavities are shown in Figure 2. The simulated efficiency is as high as $78.1 \%$. 
Comparing to the COM 08_04 04 klystron one can conclude that CSM_23 has lower level of the bunch saturation and stronger radial bunch stratification. However the compact layout with simple 5-cavity configuration makes CSM technology very attractive for the L-band klystrons. Currently we are developing next CSM tubes generation with improved performance and expected efficiency above $80 \%$.

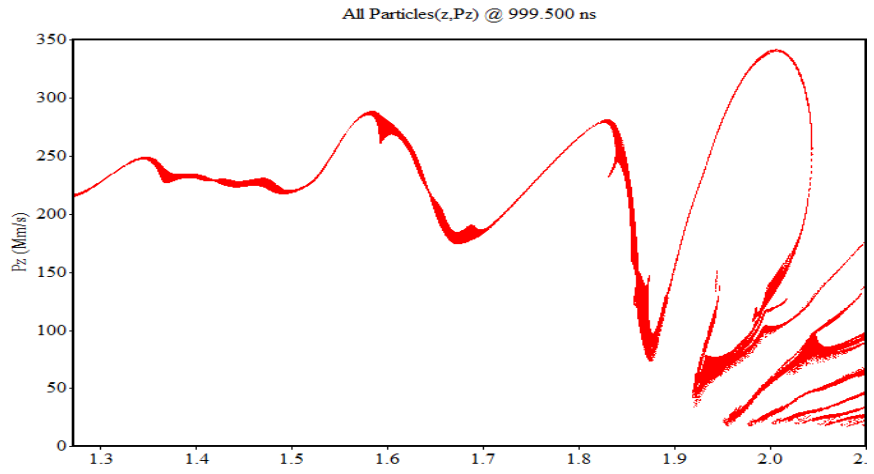

$\mathrm{z}(\mathrm{m})$

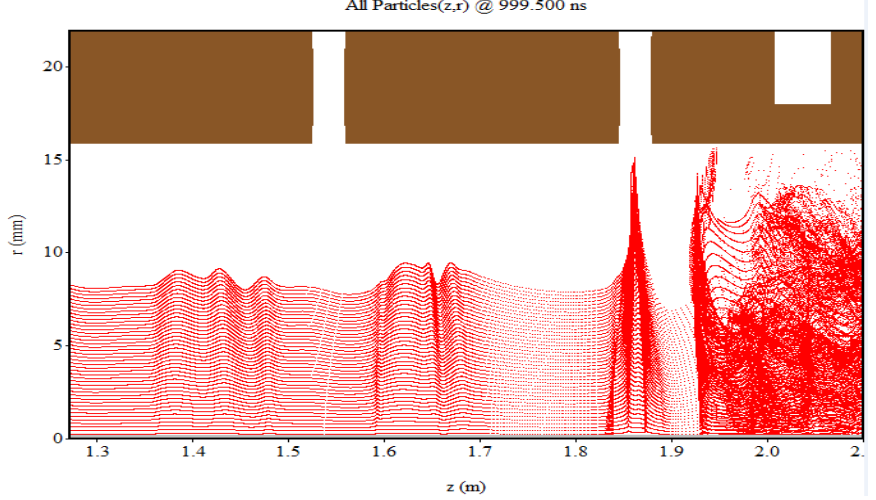

Figure 2. PZ-Z (top) and RZ (bottom) phase-space profiles of the electron beam at the final two cavities.

\section{ACKNOWLEDGMENT}

The authors would like to thank the High Efficiency International Klystron Activity (HEIKA) and associated members.

\section{REFERENCES}

[1] Aicheler, M., Burrows, P., Draper, M., Garvey, T., et al., “A Multi-TeV linear collider based on CLIC technology: CLIC Conceptual Design Report," CERN-2012-007, 2012.

[2] E. Jensen, "FCC RF overview," in First Annual Meeting of the Future Circular Collider study, 2015.

[3] Baikov, A.Yu.; Marrelli, C.; Syratchev, I., "Toward High-Power Klystrons With RF Power Conversion Efficiency on the Order of $90 \% "$ in Electron Devices, IEEE Transactions on, vol.62, no.10, pp.34063412, Oct. 2015.

[4] A. Baikov, G. Burt, D. Constable, R. Kowalcyzk, C. Lingwood, I. Syratchev, "MAGIC2-D Simulations of High Efficiency Klystrons using the Core Oscillation Method", proceeding of this conference.

[5] HE Klystron Summary Report," tech rep., C.Marrelli, European Spallation Source ESS AB, 2014.

[6] Hill, V.C.R; Marrelli, C.; Constable, D.; Lingwood, C., "Particle-in-cell simulation of the third harmonic cavity F-Tube klystron", Vacuum Electronics Conference (IVEC), 2016 IEEE International.
[7] A. J. Jensen et al., "Developing sheet beam klystron simulation capability in AJDISK”, IEEE Trans. Elec. Dev., vol. 61(6), pp. 16661671, Jan. 2014

[8] MAGIC Tool Suite, Orbital ATK, Inc, Newington, VA. [Online]. Available: http://www.orbitalatk.com/Magic/ 